\title{
Addendum to the sonographic medical act
}

\author{
Vito Cantisani ${ }^{1}\left[{ }^{10} \cdot\right.$ Giovanni lannetti $^{2} \cdot$ Vittorio Miele $^{3} \cdot$ Roberto Grassi $^{4,5} \cdot$ Michele Karaboue $^{6} \cdot$ Elviro Cesarano $^{7}$. \\ Franco Vimercati ${ }^{8} \cdot$ Fabrizio Calliada ${ }^{9}$
}

Received: 11 April 2021 / Accepted: 30 May 2021 / Published online: 9 July 2021

(c) Società Italiana di Ultrasonologia in Medicina e Biologia (SIUMB) 2021

The rapid and constant evolution of ultrasound equipment, and therefore, the possibility of using more manageable and easily transportable equipment, such as pocket ultrasound scanners, probes and devices, makes it necessary to integrate the sonographic medical act drawn up by SIUMB and SIRM with a definition of the characteristics of the sonographic examination carried out to complete the physical and medical examination.

Many synonyms have been used up to now (POCUS, bed side sonography etc.). The Societies, therefore, intend to establish rules for the sonographic examination, which is carried out to complete an internal medicine examination to answer specific questions without the need to fill in a structured report. This examination is defined here as a Sonography-Assisted Medical Examination (SAME).

The operator must be a qualified physician able to perform sonography and to investigate physical examination findings (organ volume, presence of effusion...) and to correctly interpret the classic ultrasound signs to identify what causes the most important symptoms (Mc Burney, Murphy etc.)

Vito Cantisani

vito.cantisani@uniroma1.it

Giovanni Iannetti

gianni.iannetti@virgilio.it

Roberto Grassi

presidente@fondazionesirm.org

Elviro Cesarano

elvirocesarano@hotmail.com

Franco Vimercati

francovimercati@gmail.com

Fabrizio Calliada

fabrizio.calliada@gmail.com

1 Policlinico Umberto I, Dipartimento di Scienze Radiologiche, Università La Sapienza, Rome, Italy

2 Ospedale S. Spirito, Università degli Studi Chieti-Pescara, Chieti, Italy
SAME must therefore always be performed according to the patient's clinical needs and it will constitute an integral part of the medical examination for the purpose of assisting the physician in formulating a diagnostic hypothesis thus allowing him/her to start the subsequent clinical, diagnostic, and therapeutic process.

An adequate training course organized by accredited scientific societies is required and must include theoretical as well as practical training. The intent is to provide the operators with adequate training enabling them to carry out SAME and subsequently create a 2 nd level support network with the support of Telemedicine and a network of centers of excellence to complete the diagnostic process in the shortest possible time.

As mentioned above, SAME could also be applied in a selected group of personnel (e.g., in connection with competitive entrance exams for the armed forces. where it is necessary to ensure the subject's physical fitness) and in preventive and/or occupational medicine, or in all situations where a clinical evaluation of the subject is appropriate.
3 Dipartimento di Radiodiagnostica Emergenza-Urgenza, Azienda Universitaria Careggi, Florence, Italy

4 Dipartimento di Radiologia, Università degli Studi della Campania Luigi Vanvitelli, Naples, Italy

5 Italian Society of Medical and Interventional Radiology (SIRM), SIRM Foundation, 20122 Milan, Italy

6 Dipartimento di Medicina di Precisione, Università degli Studi della Campania Luigi Vanvitelli, Naples, Italy

7 Servizio di Radiologia Centro Ospedaliero Militare Interforze - Marina Militare, Taranto, Italy

$8 \quad$ FISM - Federazione delle Società Medico-Scientifiche Italiane Villa Marelli Viale Zara, 8120159 Milano, Italy

9 Dipartimento di Radiologia, I.R.C.C.S. Policlinico S. Matteo Pavia, Università degli Studi di Pavia, Pavia, Italy 
When a portable ultrasound scanner consisting of compatible probes and monitors (e.g., tablets) is used, it is necessary that:

- the probes are certified as medical devices;

- the monitor must be suitable for medical examinations at least in terms of size, resolution and safety in accordance with the conditions in which the scanning is carried out;

- adequate internet performance.
Publisher's Note Springer Nature remains neutral with regard to jurisdictional claims in published maps and institutional affiliations. 